\title{
ACESSO A SERVIÇOS DE SAÚDE: olhar de usuários de uma unidade de saúde da famíliaa
}

Áurea Christina de Paula CORRÊA ${ }^{b}$, Flávia FERREIRAc, Graziela Souza Pinto CRU Zd ', Inês de Cássia F ranco PED ROSA ${ }^{e}$

\section{RESUM 0}

0 acesso aos serviços de saúde é um direito de todo brasileiro, e guarda relação com os princípios do acolhimento e vínculo. N este estudo de abordagem qual itativa, que tomou por referência a Carta dos Direitos dos U suários da Saúde, objetiva-se anal isar a concepção de usuários de uma U nidade de Saúde da F amília de Cuiabá, M ato G rosso, sobre acesso, acol himento e vínculo. Realizou-se um estudo de caso, sendo os dados coletados através de entrevistas semi-estruturadas, anal isados através da técnica de análise temática. Os resultados apresentam o acesso concebido de for ma nem sempre positiva devido à demora no atendimento e baixa resolutividade, induzindo a busca por outros serviços. 0 desconhecimento de seus direitos como usuários e a incipiente organização da rede de serviços explicam, em al guma medida, a percepção do usuário, apontando para a necessidade de reorganização do serviço e da própria rede, como porta de entrada do sistema.

D escritores: A cesso aos serviços de saúde. A colhimento. Programa Saúde da Família.

\section{RESUMEN}

E I acceso a los servicios de salud es un derecho de todos ciudadanos de B rasil, y está estrechamente relacionado con los principios de la atención y vinculación. E n este estudio cualitativo, se tomó por referencia la Carta de los D erechos de los U suarios de la Salud, y tiene como objetivo analizar la concepción de los usuarios de una U nidad de la Salud de F amilia de Cuiabá, M ato Grosso, B rasil, sobre el acceso, alojamiento y de bonos que habían experimentado en los servicios de salud. Se realizó un estudio de caso, con los datos recogidos a través de entrevistas semi- estructuradas y analiz ados mediante la técnica del análisis temático. L os resultados muestran que el acceso que está diseñado no siempre es positivo, debi do a la demora en el tratamiento y no a la solución de la unidad, lo que provocó la búsqueda de otros servicios que satisficieran sus necesidades inmediatas de salud. EI no conocer sus derechos como una organización incipiente y los usuarios de la red de servicios explican, en cierta medida, la insatisfacción del usuario, que apunta a la necesidad de reorganización de la unidad y la propia red, como puerta de entrada del sistema.

D escriptores: Accesibilidad a los servicios de salud. A cogimiento. Programa de Salud F amiliar. Título: A ccesibilidad a los servicios de salud: lo mirar de los usuarios de una unidad de la salud de la familia.

\section{ABST RACT}

Theaccess to health serviceis a right of every $B$ razilian citizen, and it is closely related to the principles of receptiveness and bond. This qualitative study, that took the $U$ sers' Rights Charter as a reference, aims to analyze how users from a Family $\mathrm{H}$ ealth U nit in the city of Cuiabá, state of M ato G rosso, B razil, view access, receptiveness and bond. A case study was car ried out and the data were collected through semi-structured interviews and inter preted through thematic analysis. The results show access perceived in a not alw ays positiveway, due to del ays in treatment and a low resolution of cases, leading to the search for other services. The lack of knowledge by the user of his rights and the incipient organization of the service netw ork explain, to some extent, the user's dissatisfaction, pointing to the need for the reorganization of the services and the netw ork, as they are the gatew ay to the system.

D escriptors: $\mathrm{H}$ ealth services accessi bility. U ser embracement. F amily $\mathrm{H}$ ealth P rogram.

$T$ itle: Access to health services: how users from a famil y health unit view it.

\footnotetext{
a T rabal ho desenvolvido em 2009 no contexto da disciplina T rabalho de Conclusão de Curso do Curso de G raduação em Enfermagem da U niversidade Federal de M ato Grosso (UFM T).

b D outora em Enfermagem, Coordenadora do Programa de Pós-G raduação: M estrado em Enfermagem da UF M T, Cuiabá, M ato G rosso, Brasil.

c Enfermeira Assistencial do Hospital Santa Rosa, Cuiabá, M ato Grosso, Brasil.

¿ Enfermeira da A tenção Básica do município de Chapada do Guimarães, M ato Grosso, Brasil.

e M estre em Enfermagem, M embro da Equipe T écnica da Coordenadoria de A tenção Básica da Secretaria de Estado da Saúde de M ato Grosso, Cuiabá, M ato G rosso, Brasil.
} 


\section{INT RODUÇÃO}

A Carta dos Direitos dos U suários da Saúde (CDUS), elaborada pelo Conselho $\mathrm{N}$ acional de Saúde e M inistério da Saúde (M S), baseia-se em princípios de cidadania visando garantir à população a possibilidade de conhecer seus direitos como usuários do Sistema Ú nico de Saúde (SU S). A Carta tem por princípio primeiro garantir a todo cidadão brasileiro a facilidade de acesso aos serviços de saúde do SU S, assim como às instituições conveniadas a ele. Evidencia também que, todo cidadão tem direito a receber tratamento adequado, de forma a resolver suas necessidades de saúde, ressal tando a importância de oferecer atendimento humanizado, acolhedor e livre de qualquer discriminação(1).

A CDUS tem como eixos norteadores 0 acesso e 0 acolhimento, concebidos como a entrada do usuário no Sistema de Saúde (SS) através dos vários serviços existentes na rede, pautadas no respeito, na solidariedade, no reconhecimento dos direitos e no fortalecimento da autonomia dos usuários, trabalhadores e gestores da saúde ${ }^{(2)}$.

Conceber 0 acesso e 0 acolhimento como princípios do SUS favorece a obtenção de melhores resultados nas intervenções em saúde, possibilitando a continuidade do cuidado em qualquer nível de atenção por serem elementos essenciais à assistência, uma vez que visam à qualificação do cuidado prestado.

Portanto, assumir o acesso aos serviços de saúde como direito de cidadania e necessidade incontestável leva a reconhecer que a configur ação dos modelos assistenciais de saúde deve ser definida pela organização da produção de serviços a partir de um determinado arranjo de saberes, bem como de projetos de construção de ações sociais específicas, como estratégias políticas de determinados agrupamentos sociais $s^{(3)}$.

A o tomar como referência os princípios do SU S reconhece-se que, toda instituição de saúde tem o compromisso de atender com qualidade técnica e de forma resolutiva às necessidades de saúde do usuário, reconhecendo o acesso e 0 acolhimento como fundamentais em qualquer instituição de saúde e, principalmente, nas unidades do Prog rama Saúde da Família (PSF).

A E stratégia Saúde da Família (E SF ), enquanto estratégia de reorganização da Atenção Básica à Saúde (ABS), prevê a criação de equipes multipro- fissionais responsáveis por determinado território, atuando num espaço reconhecido por sua população adscrita como porta de entrada para o SS. Suas práticas devem ter caráter multidisciplinar e ser centradas no usuário/ família, sendo necessário o desenvolvimento da capacidade de acolhê-los, responsabilizando-se pelo indivíduo e grupo familiar, visando à resolução de seus problemas saúde e promovendo sua autonomia.

Tal organização do serviço visa que 0 acesso não se configure pelo número de portas de entrada existentes na rede ou pelo volume de demanda, mas também pela qualidade do cuidado prestado(3).

Assim, tomando acesso como um princípio fundamental da ABS, o presente estudo objetiva analisar a percepção de usuários de uma U nidade de Saúde da Família (USF) do SUS em Cuiabá, M ato G rosso, acerca da organização do serviço de saúde do município, identificando em que medida os princípios do acol himento, vínculo e acesso têm se concretizado na prática daquele serviço de saúde.

\section{MET ODOLOGIA}

Trata-se de uma pesquisa qualitativa, realizada em uma USF, da Regional Norte, do município de Cuiabá, M ato G rosso. Para a coleta de dados foi usada a técnica de entrevista semiestruturada e para a análise dos dados levantados a T écnica de A nálise de Conteúdo do tipo Temática, visando identificar a percepção dos usuários acerca do acesso, acolhimento e vínculo realizados na unidade ${ }^{(4)}$.

Os sujeitos que participaram deste estudo foram selecionados com a utilização dos seguintes critérios de inclusão: ser membro de uma família moradora da área de abrangência da U SF onde 0 estudo foi realizado há mais de um ano; ter buscado atendimento para suas necessidades de saúde na unidade por pelo menos uma vez; estar apto a responder perguntas de cunho subjetivo. Assim tomaram parte do estudo sete usuários da U SF.

Os participantes foram, inicialmente, informados sobre os objetivos do estudo e a razão de sua realização, para posteriormente assinarem o T ermo de Consentimento L ivre e E sclarecido de participação nesta pesquisa, sob a garantia de sigilo acerca das informações fornecidas. 0 estudo atendeu os princípios legais estabelecidos pela Resolução 196/ 96 do Conselho N acional de Saúde, que regulamenta a realização de pesquisas en- 
volvendo seres humanos ${ }^{(5)}$, e obteve aprovação final para sua realização do Comitê de Ética e Pesquisa (CEP) do Hospital U niversitário Julio M uller (HUJM ), sob protocolo no 559/ CEP-HUJM / 08.

As entrevistas ocorreram individualmente, em local e horário estipulados pelos entrevistados. Os entrevistados foram indagados sobre: 0 local (serviço) que buscam para solucionar seus problemas/ necessidades de saúde; a forma como são recebidos pelos profissionais da equipe da USF do estudo; as dificuldades que identificam/ percebem para conseguirem 0 atendimento demandado; se a equipe da USF contribui para a resolução de seus problemas de saúde e do bairro. As entrevistas foram gravadas em fita K-7 e, posteriormente, transcritas para realização da análise, e identificadas da seguinte forma: E 1; E 2, E 3, E 4, E 5 e E 6 .

Os dados foram analisados a partir das categorias pré-definidas: Percepção dos usuários acerca do acesso aos serviços de saúde; 0 acolhimento da comunidade pela equipe da USF e a formação de vínculo e A integralidade e a resolutividade da assistência. Para a discussão dos dados tomou-se por referências os princípios do SUS - acesso, acoIhimento e vínculo - segundo discussão teórica veiculada nacionalmente.

\section{RESULT ADOS E DISCUSSÃO}

\section{Percepção dos usuários acerca do acesso aos serviços de saúde}

Segundo o M S, a ABS deve configurar-se como porta de entrada preferencial para o $\mathrm{SS}^{(2)}$ que, se bem organizada, tem potencial para resolver/ atender a maioria dos problemas/ necessidades de saúde da população, filtrando o acesso a níveis de maior complexidade de assistência através da realização de encaminhamentos para a atenção especializada, da utilização do serviço sempre que necessário e a exigência do atendimento, assegurando seus direitos ${ }^{(6)}$.

0 acesso à saúde deveria extrapolar a assistência em si, sendo a acessibilidade aos diversos níveis de complexidade dos serviços fundamental para a concretização desse direito universal(7), quando necessário, de forma rápida e oportuna.

E mbora na USF de referência dos sujeitos deste estudo 0 atendimento clínico ocorra de forma rotineira, al guns entrevistados não o consideram adequado, em função da espera prolongada, deter minando a busca de outros serviços para resolução do problema/ necessidade de saúde eminente, como nos excertos que seguem.

[ ...] ai chegué lá tem um monte de gente que foi adiado porque o médico tinha que fazer não sei o que lá, e eu não sei, vim embora não consultei ... (E 3).

[...] quando num tem atendimento a gente tem que ir pra outro lugar [ ...] Aqui é muitíssimo complicado. [...] Porque sempre que você vai marca um exame, às vez es pode até marcar, mas é daqui uns dois meses, três meses, entendeu? (E 5).

Ao se tomar por referência as normativas do M S concebe-se que a não garantia da manutenção da ABS como porta de entrada de fácil acesso, disponível e resolutiva para 0 atendimento às necessidades de saúde da população viola o direito do usuário(1) e compromete assistência adequada, por vezes postergando sua ocor rência, afetando negativamente o diagnóstico e o manejo do problema, resultando na busca do serviço de saúde em outro nível de atenção(6).

Entretanto, apesar da espera prolongada, alguns usuários concebem que suas necessidades de saúde são atendidas, considerando a proximidade da U SF com a comunidade.

0 posto desaúde [ ...] E m qualquer mal-estar queaparece a gente vai lá. [ ...] 0 atendimento é rápido também (E 2).

0 posto de saúde [...] a gente procura lá primeiro, dependendo do horário, mas sefor a noite vai na policlínica. Sempre quando a gente precisa... sempre foi bom... fora que não é longe (E 6).

As falas apresentadas demonstram diferentes percepções acerca do acesso a U SF, entretanto, estudiosa da ABS no país aponta o acesso como a primeira etapa a ser vencida pelo usuário na busca da satisfação de suas necessidades de saúde ${ }^{(8)}$, devido a restrição do horário de funcionamento e a indisponibilidade de horários alter nativos, o que deter mina a busca de outros serviços.

Frente ao exposto, é preciso considerar que, no que diz respeito a garantia do acesso ao serviço de saúde, para além do estabelecido pelo M S, a organização da ABS requer a articulação entre o normatizado, o contexto municipal e o preparo/ disponibilidade da equipe para implementação da 
proposta de reorganização do modelo assistencial.

E $m$ função de tal organização da rede, segundo os sujeitos deste estudo, é necessário buscar respostas mais rápidas em outros serviços quando não há resolutividade local. A ssim, al guns usuários relataram terem deixado de buscar atendimento as suas necessidades na U SF referência.

[ ...] eu nem procurava mais, nem para medir pressão, nem pra nada, já ia no HUJ M, entendeu? [ ...] eu não fui nesse médico aí ainda... (E 3).

Para fazer CD da minha filha, tive que parar uns tempos, agora, porque não tava atendendo né, e então eu não procuro (E 3).

As falas evidenciam que a unidade tem garantido a acessibilidade de maneira parcial, uma vez que, a assistência nem sempre é resolutiva, fator que interfere na escolha do sujeito na busca pelo serviço de saúde. V isando a resolução de seus problemas, usuários lançam mão de relações pessoais para facilitar sua entrada no SS, o que favorece seu acesso aos serviços.

[ ...] falaram pra mim que eu tinha que ir lá no H U J M. [ ...] aí minha cunhada trabalha lá falou que eu posso ir, [ ... fazer da neném, tratamento da nenê, tudo pra lá, porque não adianta fica aqui (E 3).

[ ...] minha esposa é técnica de enfermagem, ai ocorre "aquele conhecimento" né, ai a gente procura um médico que ela conhece há bastante tempo né, [ ...] e problema resolvido (E 1).

A titudes deste tipo determinam desigualdades no acesso aos serviços de saúde, contribuindo com a iniqüidade, uma vez que não há garantia de igualdade de oportunidade de acesso(9). Tal atitude é compreensível na medida em que o sistema não responde às necessidades de saúde da população com a agilidade necessária.

A baixa resolutividade local gera encaminhamentos diversos aos serviços de referência.

E u procuro o posto né? [ ...] "N ão temos médico, tem que ir pro P ronto Socorro ou então para a policlínica, sinto muito". [ ...] Ah!! M édico nunca quase tem, né? (E3).

[ ...] ele falou que o meu filho tava muito com febre, então el enão atendeu, [ ...] então eu fui lá no P lanal- to; sempre no Planalto, ou então lá na policlínica do Verdão (E 4).

Refletir sobre resolutividade na correlação com 0 acesso também requer que se considere 0 processo organizativo proposto para o trabalho na ESF, uma vez que se prevê a realização de ações em equipe multiprofissional, fundamentada no diálogo, respeitando-se a competência de cada profissional ${ }^{(10)}$. T rabal ho esse que deve ser desenvolvido com vistas a humanização da assistência, por profissionais tecnicamente competentes, com ações intersetorialmente articuladas e socialmente apropriadas $^{(11)}$.

Tomando por referência as falas dos sujeitos deste estudo, obser va-se que a ausência de um dos membros da equipe prejudica, em alguma medida, a resolutividade da USF estudada, induzindo o usuário à busca de outros serviços, o que compromete a acessibilidade e viola os direitos do usuário.

\section{0 acolhimento da comunidade pela equipe da USF e a formação de vínculo}

Conceitualmente 0 acolher relaciona-se à implementação de atitudes humanizadas, como o saber ouvir demonstrando interesse em resolver os problemas apresentados, esgotando as possibilidades de solucionar as necessidades apresentadas pelos usuários ${ }^{(12)}$.

0 exercício cotidiano do acol himento possibilita a captação das necessidades de saúde manifestas pelo usuário, refletindo-se em um processo de trabalho concretizado em ações que respondam às necessidades levantadas, favorecendo a acessibilidade aos serviços de saúde ${ }^{(13)}$.

$\mathrm{N}$ esse sentido, 0 acolhimento tem papel fundamental na assistência à saúde, pois visa 0 atendimento ético, humanizado e cidadão, como forma de receber e atender usuários no âmbito dos serviços de saúde, favorecendo a interação entre equipe e população(14).

Entretanto, os excertos que seguem apresentam uma dualidade, pois evidenciam 0 acolhimento se efetivando nas práticas cotidianas através da assunção de posturas éticas e, subliminarmente denuncia ausências ao serviço, gerando prejuízos ao atendimento acolhedor.

[ A enfermeira] [ ...] ela pode ta atendendo quem for, ela chega e fala "espera só um pouquinho que vou te 
atender". E la vem de lá me atende, se precisar medir minha pressão ela manda as mulher medir (E 3).

[...] eu procuro a E nfermeira, a gente sempre fala com a E nfermeira [ ...] Às vezesa gentechega lá efala: "eu preciso, quenão sei o que; [ ...] Ah, não tem médico, não sei o que"; ai a gente vai direto na E nfermeira, daí da vê se pode ajuda ou não... a gente só vê mais é com ela (E 6).

Segundo as falas, 0 acolhimento está presente em ações específicas do enfermeiro, no entanto, acolher é uma ação inerente a equipe, um direito do usuário e um princípio do SUS, onde "humanizar" as relações entre usuários e trabalhadores significa compreender que os sujeitos são dotados de desejos e necessidades ${ }^{(1,15)}$.

Para além, 0 acolhimento se traduz na organização do serviço de saúde para receber e responder as demandas, de forma a atender o usuário lhe garantido a acessibilidade; reorganizando o processo de trabal ho e qualificando a relação trabaIhador/ usuário a partir de parâmetros humanitários de solidariedade e de cidadania ${ }^{(15,16)}$.

0 trabalho no PSF deve ser implementado pela equipe em conformidade com os níveis de competência, de acordo com a capacidade de resolução de cada membro, tomando por referência a complexidade dos problemas e necessidades de saúde dos indivíduos e da coletividade ${ }^{(16)}$.

A efetivação do acolhimento possibilita a criação de vínculos, favorecendo as reações dos usuários acerca das informações recebidas, seu entendimento e observâncias sobre suas necessidades, determinando ou não a satisfação com a atenção recebida ${ }^{(17)}$.

Com vistas à criação de vínculo são realizadas Visitas D omiciliares (VD) pela equipe de referência de forma regular na comunidade.

[ ...] já gosto de que, que eles vem aqui. [ ...] [ a enfermeira] vem pouco também. Vem. Q uando énas épocas de vacina, elas vêm pouco aqui (E 1 ).

É o médico já veio aqui uma vez, éuma vez ele já veio, o médico, foi a enfermeira e só também. [ ...] A agente de saúde semprevem, sempre, semprequase toda semana ela vem aqui (E2).

A VD é uma tecnologia voltada para efetivação do acolhimento e fortalecimento do vínculo, sendo, via de regra, realizada pelo ACS que deve manter contato permanente com as famílias da área adscrita ${ }^{(2)}$, fortalecendo o vínculo entre serviço e população, reforçando a importância de seu papel na equipe.

As VD, no PSF, também devem ser realizadas pelo médico e enfermeiro quando houver indicação ou necessidade constatada, e rotineiramente pelo ACS, otimizando-se a utilização de recursos humanos e evitando a dependência da população dos profissionais de saúde ${ }^{(18)}$.

Entretanto, no cotidiano da USF deste estudo a VD não é regularmente realizada por todos profissionais da equipe, contrariando a proposta da ESF, o que se justifica pela forma de organização do trabal ho assumida na unidade, proveniente do contexto local.

Refletir sobre acolhimento e vínculo no contexto da U BS deste estudo requer a ampliação para além do prescritivo, considerando que não seja possível negar que influências exter nas são determinantes na organização da U SF tomando por referência o contexto local, ou seja, a equipe possui uma identidade própria que pode ou não estar voltada a concretização de um novo modelo assistencial, e sofre influencia direta da organização da rede municipal de saúde, e de fatores que determinam fortemente a forma de agir dos profissionais envolvidos na assistência ao usuário.

\section{A integralidade e a resolutividade da assistência}

A integralidade pressupõe um conjunto de ações contínuas e de serviços, que visam gar antir promoção, proteção, cura, reabilitação dos usuários, e de ações intersetoriais de promoção da saú$\mathrm{de}^{(19)}$. E ntretanto requer que a equipe reconheça a variedade de necessidades do paciente e disponibilize recursos para atendê-las, quando necessário e em todos os momentos do ciclo de vida do usuário ${ }^{(6)}$.

A integralidade das ações depende da acessibilidade aos serviços. A falta de acesso compromete a finalização do cuidado e a gar antia da resolutividade dos problemas dos usuários ${ }^{(12)}$. N esse sentido, alguns sujeitos deste estudo mostram-se conformados com a baixa resolutividade.

[ ...] as vezes não é da competência pra eles resolver as vez es eles levam lá, mas lá do outro lado lá que enrola o problema. [ ...] Q uestão de encaminhamento [ ...] I sso, esse que é o problema maior (E 1$)$. 
Quando é al guma coisa assim que eles não resolve aqui. Aí el es, encaminha vocêpra outra unidade. [ ...] quando é pra você marca um outro exame, em outra unidade, aí que é demorado [ ...] (E 5).

[...] eles tenta, eles tenta faz consulta, examina tudo, conforme seja o caso el es faz a ficha manda a gentepra polidínica, assim queé... (E 2).

As falas demonstram a insatisfação com a demora nos encaminhados aos serviços de referência. No entanto, para a U SF ser resolutiva precisa estar articulado a uma rede de serviços com outros níveis de atenção, além de depender de relações intersetoriais $s^{(6,10)}$.

Algumas falas evidenciam a unidade como cumpridora de seu papel de ordenadora do cuidado, buscando a resolução do problema em outros níveis de atenção.

No entanto, quando as necessidades de saúde não são atendidas em tempo hábil, buscam atendimento em serviços de maior complexidade, desconsiderando o fluxo das referências, o que gera descrédito do serviço e dos profissionais da saúde.

[ ...] sabe onde é que eu to fazendo tratamento de minha vista? Lá em Várzea G rande, no posto de Várzea Grande, eu moro aqui em Cuiabá, cheguei lá marcaram a consulta pra mim, consegui consultar com o me Ihor médico da vista $D r$. F e aqui ninguém conseguiu marcar médico pra mim (E 3).

0 excerto demonstra certa fragilidade da estrutura da rede assistencial, contrariando a função de porta de entrada para o SS, com garantia de acesso e cuidado integ ral ${ }^{(2)}$.

A demais, persiste a cultura de chegar cedo para assegurar atendimento médico, evidenciando que, organizacionalmente, a instituição da ESF determinou poucas mudanças no cenário da unidade.

[...] ol ha eu já vi gente amanhecer aí já, eu já vi gente 5 horas da manhã, 4 horas da manhã ta lá na fila já porque tem uma quantidade $X$ de pessoas queelesatendem né, num é assim vai chegando e vai atendendo, é tipo assim 10 a 20 pessoas que eles atende (E 1 ).

Os excertos demonstram que a organização da unidade, neste aspecto, ainda favorece a concepção do modelo biomédico, caracterizada pela medicalização da assistência, que se mantém ainda hegemônica ${ }^{(17)}$.
O u seja, para a consolidação da proposta da ESF, com vistas ao atendimento à saúde daquela população, mudanças precisam ocorrer para que 0 direito à saúde se efetive como direito de cidadania. $\mathrm{N}$ esse sentido, cabe aos gestores a reestruturação da rede assistencial e às equipes das USF, como empreendedoras da mudança do modelo e promotoras de cidadania, promoverem ações que favoreçam 0 acesso da população ao SS ${ }^{(20)}$.

\section{CONSIDERAÇÕES FINAIS}

A realização deste estudo per mitiu a constatação de que, para os sujeitos desta pesquisa, 0 acesso a U SF que serve de referência para suas famílias car acteriza-se pela demora no atendimento, pelo absenteísmo e pela baixa capacidade resolutiva, fatores que levam o usuário a não retor nar a unidade, buscando a atenção secundária como porta de entrada preferencial para o SS e resolução de suas necessidades.

Os dados apontam a U SF de referência como ordenadora do cuidado, devido à proximidade da unidade da residência dos usuários, possibilitando a ampliação do acesso ao SS.

A análise realizada aponta para a existência de práticas que favorecem iniqüidades no acesso, uma vez que alguns usuários lançam mão de práticas alternativas para aquisição de benefícios na busca de atendimentos facilitados em outros serviços, enquanto a maioria dos usuários do SU S depende da agilidade do sistema.

A postura assumida pela enfer meira, relatada pelos usuários, favorece o acolhimento e a criação de vínculo com os usuários, entretanto, as VD são implementadas, na maioria das vezes, pelas ACS que a realizam regularmente.

Frente ao exposto é possível considerar que, U SF referência dos sujeitos desta pesquisa encontra-se em processo de consolidação da ESF, mas ainda precisa reaval iar seus processos inter nos com vistas a al cançar al inhamento entre a organização da unidade, a rede de serviços de saúde no município e a proposta ministerial.

\section{REFERÊ NCIAS}

1 M inistério da Saúde (BR ), Consel ho N acional de Saúde. Carta da 11ā Conferência Nacional de Saúde. In: Relatório final da 11a Conferência N acional de Saúde: efetivando o SU S: acesso, qual idade e humanização na 
atenção à saúde com controle social; 2000 dez 15-19; Brasília (DF), Brasil [ Internet] . Brasília (DF); 2001 [ citado 2009 maio 18]. Disponível em: http:/ / bvsms.saude.gov.br/ bvs/ publicacoes/ 11_cns.pdf.

2 M inistério da Saúde (BR), Secretaria de Atenção à Saúde. Portaria 648/ G M , de 28 de março de 2006: aprova a Política de A tenção Básica, estabelecendo a revisão de diretrizes e nor mas para a organização da A tenção Básica e para o Programa Saúde da Família (PSF ). Brasília (DF); 2006.

3 M erhy EE, M agalhães JH M, Rimoli J, Franco T B, Bueno W S. 0 trabalho em saúde: olhando e experienciando o SU S no cotidiano. 2a ed. São Paulo: H ucitec; 2006.

4 G omes R. A análise de dados em pesquisa qual itativa. In: M inayo M CS, organizadora. Pesquisa social: teoria, método e criatividade. Petrópolis: Vozes; 2001. p. 67-80.

5 M inistério da Saúde (BR ), Consel ho N acional de Saúde. Resolução 196, de 10 de outubro de 1996: diretrizes e normas regulamentadoras de pesquisa envolvendo seres humanos. Brasília (D F ); 1996.

6 E scorel S, Giovanella L, M agalhães M HM, Senna M CM . O Programa de Saúde da F amília e a construção de um novo modelo para a atenção básica no Brasil. Rev Panam Salud Publica. 2007;21(2/ 3):164-176.

7 U nglert CV S. 0 enfoque da acessibilidade no planejamento da local ização e dimensão de ser viços de saúde. Rev Saúde Pública. 1990;24(6):445-52.

$8 \mathrm{M}$ atumoto S. 0 acolhimento: um estudo sobre seus componentes e sua produção em uma unidade da rede básica de serviços de saúde [ dissertação]. Ribeirão Preto: E scola de Enfermagem de R ibeirão Preto, U niversidade de São Paulo; 1998.

9 Sousa M F. A cor-agem do PSF. São Paulo: Hucitec; 2001.

10 Peduzzi M . Equipe multiprofissional de saúde: conceito etipologia. Rev Saúde Pública. 2001;35(1):103-9.

\section{Endereço da autora / Dirección del autor / Author's address:}

Á urea Christina de Paula Cor rêa

Av. M arechal D eodoro, 829, ap. 103, Araes

78000-100, Cuiabá, M ato G rosso

E-mail: aureaufmt@gmail.com
11 Fracolli LA, Zoboli E LCP. Descrição e análise do acolhimento: uma contribuição para o Programa de Saúde da F amília. Rev E sc E nferm U SP. 2004;38(2): 143-51.

12 T akemoto M LS, Silva E M . A colhimento e transformação no processo de trabalho de enfermagem em unidades básicas de saúde de Campinas, São Paulo, Brasil. Cad Saúde Pública. 2007;23(2):331-40.

13 Franco T B, Bueno W S, M erhy E E . 0 acolhimento e os processos de trabal ho em saúde: 0 caso de Betim, M inas G erais, Brasil. Cad Saúde Pública. 1999;15(2): 345-53.

14 T eixeira CF. A mudança do model o de atenção à saúde no SU S: desatando nós, criando laços. Saúde D ebate. 2003;27(65):257-77.

15 Souza AC, L opes M J M . A colhimento: responsabilidade de quem? U m relato de experiência. Rev G aúcha Enferm. 2003;24(1):8-13.

16 Colomé ICS, D avis S, L ima M ADS. V isão de enfermeiras sobre as articulações das ações de saúde entre profissionais de equipes de saúde da família. Rev E sc Enferm U SP. 2008;42(2):256-61.

17 Starfield B. Atenção primária: equilíbrio entre necessidades de saúde, serviços e tecnologia. Brasília (DF): U N E SCO/ M inistério da Saúde; 2004.

18 M inistério da Saúde (BR), Secretaria de A ssistência à Saúde, Coordenação de Saúde da Comunidade. Saúde da família: uma estratégia para reorientação do modelo assistencial. Brasília (DF ); 1997.

19 Vasconcelos CM, Pasche DF. O sistema Ú nico de Saúde. In: Campos G W, organizador. T ratado de saúde coletiva. São Paulo: Hucitec; 2006. p. 531-62.

20 Ferreira F, Cruz G SP. A cesso a serviços de saúde: olhar do usuário [ monografia] . Cuiabá-M T : F aculdade de E nfermagem, $\mathrm{U}$ niversidade Federal de $\mathrm{M}$ ato Grosso; 2009.

Recebido em: 04/ 10/2010

A provado em: 30/ 08/ 2011 